18 YouTube: http://www.youtube.com/watch?v= c17cIyO3S4Q (Zugriff: 01.05.2011, 17.40 Uhr).

19 YouTube: http://www.youtube.com/watch?v= lBuRkBVdF8o (Zugriff: 30.04.2011， 16.36 Uhr).

20 YouTube: http://www.youtube.com/watch?v= 1l6ALmQcG_A (Zugriff: 01.05.2010, 10.40 Uhr).

21 YouTube: http://www.youtube.com/watch?v= zoFVpGXXt9g (Zugriff: 01.05.2010, 17.10 Uhr).

22 YouTube: <UNTER>http://www.youtube.com/ watch?v=HPvk4_GpP8w<unter> (Zugriff: 01.05.2011, 15.35 Uhr).

23 Die wissenssoziologische Hermeneutik ist die sozialwissenschaftliche Variante der „Kunstlehre des Verstehens“. Vgl. Raab, Jürgen: Visuelle Wissenssoziologie, Theoretische Konzeption und materiale Analysen, UVK Verlagsgesellschaft mbH, Konstanz 2008, S. 134.

24 Vgl. Raab, Jürgen: Visuelle Wissenssoziologie, Theoretische Konzeption und materiale Analysen, UVK Verlagsgesellschaft $\mathrm{mbH}$, Konstanz 2008, S. 156.

25 Vgl. Raab, Jürgen: Visuelle Wissenssoziologie, Theoretische Konzeption und materiale Analysen, UVK Verlagsgesellschaft $\mathrm{mbH}$, Konstanz 2008, S. 152.

26 Die Einstellung ist die kleinste kontinuierlich belichtete filmische Einheit, die jeweils mit einem Schnitt beginnt bzw. endet. Vgl. Korte, Helmut: Einführung in die Systematische Filmanalyse, Ein Arbeitsbuch, 4. Auflage, Erich Schmidt Verlag GmbH \& Co. KG, Berlin 2010 S. 34 .

27 Vgl. Reichertz, Jo/Englert, Carina Jasmin: Einführung in die qualitative Videoanalyse, Eine hermeneutisch-wissenssoziologische Fallanalyse, VS Verlag für Sozialwissenschaften, Wiesbaden 2011, S. $14 \mathrm{f}$.

28 Vgl. Korte, Helmut: Einführung in die Systematische Filmanalyse, Ein Arbeitsbuch, 4. Auflage, Erich Schmidt Verlag GmbH \& Co. KG, Berlin 2010, S. 37

29 Vgl. Faulstich, Werner: Grundkurs Filmanalyse, 2. Auflage, Wilhelm Fink Verlag, Paderborn 2008, S. $121 \mathrm{ff}$.
30 Vgl. Berger, Peter L./Luckmann, Thomas: Die gesellschaftliche Konstruktion der Wirklichkeit, Eine Theorie der Wissenssoziologie, 23. Auflage, Fischer Taschenbuchverlag GmbH, 2010, S. $21 \mathrm{ff}$.

31 Vgl. Mayring, Philipp: Qualitative Inhaltsanalyse, Grundlagen und Techniken, 11. Auflage, Beltz Verlag, Weinheim und Basel 2010.

$32 \mathrm{Vgl}$. Kuckartz, Udo: Einführung in die computergestützte Analyse qualitativer Daten, 3., aktualisierte Auflage, VS Verlag für Sozialwissenschaften, Wiesbaden 2010, S. $12 \mathrm{ff}$

33 YouTube: http://www.youtube.com/watch?v= lBuRkBVdF8o (Zugriff: 30.04.2011, 16.36 Uhr).

34 Vgl. Reichertz, Jo/Englert, Carina Jasmin: Einführung in die qualitative Videoanalyse, Eine hermeneutisch-wissenssoziologische Fallanalyse, VS Verlag für Sozialwissenschaften, Wiesbaden 2011, S. 27.

35 Die sogenannte 89-10-1-Regel besagt, dass etwa 89 Prozent der Internetnutzer niemals und 10 Prozent gelegentlich interagieren. Ein Prozent der Nutzer produziert und veröffentlicht Inhalte. Vgl. Arthur, Charles: What is the $1 \%$ rule? The Guardian (Technology section), Thursday 20 July 2006, verfügbar unter: http:// www.guardian.co.uk/technology/2006/jul/20/ guardianweeklytechnologysection2 (Zugriff: 09.05.2011, 11.20 Uhr). Nach Gscheidle und Fisch stellen sieben Prozent der Nutzer von Videoportalen selbst Informationen ein. Vgl. Gscheidle, Christoph/Fisch, Martin: Onliner 2007: das "Mitmach-Netz" im Breitbandzeitalter. PC-Ausstattung und Formen aktiver Internetnutzung: Ergebnisse der ARD/ZDF Online-Studie 2007, in: Media Perspektiven, 2007, Nr. 8, S. 393 bis 406, S. 401. Eine andere Untersuchung ergab, dass 13 Prozent der Nutzer von Videoplattformen Kommentare hochladen. 25 Prozent davon sind zwischen 18 und 29 Jahren, neun Prozent zwischen 30 und 49 und fünf Prozent sind zwischen 50 und 64 Jahren. Vgl. Madden, Mary: Pew/Internet \& American Life Project, Online Video, 25. Juli 2007, verfügbar unter: http://www.pewin ternet.org/ /media//Files/Reports/2007/ PIP_Online_Video_2007.pdf.pdf (Zugriff: 02.06.2011, 17.20 Uhr), S. III.
36 Vgl.Google,doubleclickadplanner:https://www. google.com/adplanner/planning/site_profile ?hl=de\#siteDetails? identifier =youtube com\&geo=DE\&trait_type $=1 \& l p=$ true $\quad(\mathrm{Zu}-$ griff: 30.06.2011, 12.15 Uhr).

37 Vgl. Beißwenger, Achim (Hrsg.): YOUTUBE und seine Kinder, Wie Online-Video, Web TV und Social Media die Kommunikation von Marken, Medien und Menschen revolutionieren, Nomos Ed. Fischer, Baden-Baden 2010, S. 75.

38 YouTube: http://www.youtube.com/user/cdutv? ob=5 (Zugriff: 23.06.2011, 11.05 Uhr).

39 YouTube: http://www.youtube.com/user/FDP? blend $=1 \& \mathrm{kob}=5 \quad$ (Zugriff: 23.06.2011, 11.00 Uhr).

40 YouTube: http://www.youtube.com/user/SPD vision?ob=5 (Zugriff: 23.06.2011, 11.05 Uhr)

41 YouTube: http://www.youtube.com/user/GRUE NE?ob=5 (Zugriff: 23.06.2011, 11.10 Uhr).

42 YouTube: http://www.youtube.com/user/Bun deswehr?feature=pvchclk (Zugriff: 24.06.2011, 09.30 Uhr).

43 Nach Ausführungen eines leitenden Vertreters der Informations- und Medienzentrale der Bundeswehr.

44 Vgl. Buhr, Thomas de/Tweraser, Stefan: My Time is Prime Time, in: Beißwenger, Achim (Hrsg.): YOUTUBE und seine Kinder, Wie Online-Video, Web TV und Social Media die Kommunikation von Marken, Medien und Menschen revolutionieren, Nomos Ed. Fischer, Baden-Baden 2010, S. 69 bis 91, S. 73.

45 Vgl. Witte, Barbara: Journalismus - Partizipation - Öffentlichkeit: Das Social Web in der Politikberichterstattung, in: Zerfaß, Ansgar/ Welker, Martin/Schmidt, Jan (Hrsg.): Kommunikation, Partizipation und Wirkungen im Social Web, Band 2 Strategien und Anwendungen: Perspektiven für Wirtschaft, Politik und Publizistik, Herbert von Halem Verlag, Köln 2008 , S. 97 bis 115 , S. 109.

46 Vgl. Bundesministerium des Innern (Hrsg.): Polizeiliche Kriminalstatistik 2010, IMK Kurzbericht, Bundesministerium des Innern, Berlin 2011, verfügbar unter: http://www.bka.de/pks/ pks2010/download/pks2010_imk kurzbericht. pdf (Zugriff: 26.06.2011, 22.00 Uhr), S. 7 f.

\title{
Zivilcourage bei Gewaltgeschehnissen im öffentlichen Raum
}

Thomas Weber

In den 1990er-Jahren war der Begriff „Zivilcourage" eng mit dem Einschreiten gegen Rassismus und Fremdenfeindlichkeit verknüpft. Heute ist er durch Medienaufmerksamkeit auf Gewaltereignisse im öffentlichen Raum („Dominik Brunner“) ins öffentliche Interesse gerückt. Deren Stellenwert wird hervorgehoben durch eine anhand solcher Fälle wieder aufgerollte Diskussion über mangelnde Hilfsbereitschaft und der Forderung nach mehr Zivilcourage. Täter sollten -so die Forderung- mit dem
Einschreiten eines oder mehrerer Zeugen sowie mit einer später erfolgenden Bestrafung ihres Fehlverhaltens rechnen müssen. ${ }^{1}$ Neben der Verantwortung jedes Einzelnen spielen auch die Medien in ihrer Berichterstattung über Kriminalität und spektakuläre Gewaltereignisse eine gewichtige Rolle, vor allem bei entsprechenden Aufrufen zur Stärkung von Solidarität und Zivilcourage durch deren positive Darstellung (Baringhorst, 1999, 2001; zitiert nach: Meyer, 2004a, S. 44). Mittelbar unterstützend wirkt auch der Wandel der Berichterstattung aufgrund einer veränderten öffentlichen Wahrnehmung, die das Opfer stärker in den Fokus rückt als den Täter als ein „Opfer der Gesellschaft“ (Kersten, 2008, S. 297).

In der nachfolgenden Studie werden Zivilcouragefälle als Situationen verstanden, innerhalb derer sich die Hilfeleistung aus Sicht des Helfenden in Form einer Interaktion gegen einen anderen (Täter) richtet, der einen Dritten (Opfer) der Gefahr einer 
Schädigung aussetzt oder ihm bereits Schaden zufügt. Während sich Sachverhalte, die ein zivilcouragiertes Einschreiten erfordern, auf vielfältige Art darstellen können, sind die Reaktionsmöglichkeiten eines couragierten Helfers begrenzt. Dem Helfer bietet sich zunächst die Möglichkeit der Hilfeleistung, indem er zusätzliche Hilfe herbei holt, z. B. durch Absetzen eines Notrufs. Darüber hinaus kann er direkt mit dem Täter auf verbale und auch körperliche Art und Weise interagieren, wobei Helfer und Täter naturgemäß antagonistische Ziele verfolgen. Der Geschehensverlauf beim Einschreiten wird von bewusstem und unbewusstem Verhalten von Täter und Helfer sowie von situationsbedingten Faktoren beeinflusst. Fallanalysen und die Identifikation von wiederkehrenden ablaufprägenden Faktoren sollen es ermöglichen, Typen von Zivilcourage zu unterscheiden. Die Ergebnisse sollen Ansätze für die Phänomenologie von Zivilcouragefällen sowie für die Erkennung von Risikofaktoren bieten, z. B. als Warnsignale für eine drohende Eskalation.

Während sich die bisherige Forschung zum Phänomen der Zivilcourage vorwiegend mit der Frage nach den zugrunde liegenden fördernden und hemmenden Faktoren couragierten Handelns befasst hat, ${ }^{2}$ lag das Augenmerk meiner Studie auf der Analyse des Geschehensablaufs sowie der Art und Weise der Hilfeleistung und der Interaktion beginnend beim unmittelbaren Ansetzen zur Hilfeleistung. Die Ursprünge der Forschung zu Zivilcourage liegen in der Hilfeleistungsforschung, der Frage nach den Einflussfaktoren, die unterschiedliches Verhalten von Zuschauern bei Notsituationen erklären also warum manche Menschen helfen und andere dies in einer vergleichbaren Situation nicht tun (Darley \& Latané, 1968, S. 377). ${ }^{3}$ Zur Verdeutlichung der Zusammenhänge der einzelnen Faktoren haben Latané \& Darley ein „Prozessmodell für Hilfeverhalten “ entwickelt, das die psychologischen Prozesse beim Helfer darstellt und das $\mathrm{Zu}$ standekommen der Entscheidung zum Eingreifen/Nichteingreifen aufzeigt (Latané \& Darley, 1970; zitiert nach: Bierhoff, 2010, S. 117 ff., Labuhn, 2004, S. 83 ff., Oßwald et al., 2007, S. 131 ff). Die fünf Stufen des Prozessmodells verlaufen chronologisch und ergeben eine Hilfeleistung nur dann, wenn alle Sequenzen „erfolgreich“ durchlaufen wurden. Der „Bystander"Effekt beschreibt tatsächlich nur einen der Einflussfaktoren, nämlich die verringerte Wahrscheinlichkeit bzw. Schnelligkeit des Eingreifens einer Person bei einer Notsitu- ation aufgrund der Anwesenheit weiterer Zeugen (ebda., S. 377 f.). ${ }^{4}$ Die untersuchten Determinanten lassen sich in personenbezogene, situationsbedingte und soziale Faktoren unterscheiden und sind aufgrund der Nähe von Zivilcourage zur Hilfeleistung - beide Konstrukte sind Unterformen prosozialen Verhaltens (Batson, 1998, S. ${ }^{\circ 282}$ ) - auch übertragbar (Jonas \& Brandstätter, 2004, S. 187 ff.; ebenso: Oßwald, Frey, Greitemeyer \& Fischer, 2007, S. 129).

Die Unterscheidung von Hilfeleistung und Zivilcourage bedarf der Beurteilung einer vorliegenden Gefahr durch den potenziellen Helfer (Labuhn, Wagner, van Dick \& Christ, 2004, S. 94) sowie möglicher negativer Konsequenzen, ${ }^{5}$ die ihm durch das Einschreiten bzw. als Folge dieses Vorgehens entstehen können. In Fällen bloßer Hilfeleistung besteht für einen Helfer nur geringe bzw. keine Gefahr negativer sozialer ${ }^{6}$ Konsequenzen (Fischer, Greitemeyer, Pollozek \& Frey, 2006, S. 91), vielmehr wird ihm regelmäßig Lob und Anerkennung für sein Tätigwerden zuteil werden. Im Unterschied zur Hilfeleistung ${ }^{7}$ zeichnen sich Zivilcouragesituationen durch eine triadische soziale Konstellation von Opfer, Täter und dem couragierten Helfer aus, wobei das Opfer dabei nicht zwangsläufig anwesend sein muss. ${ }^{8}$ ein Geschehen, das sich in der Öffentlichkeit abspielt (Meyer, 2004b, S. 37) und in dessen Verlauf zentrale Wertüberzeugungen oder die Integrität ${ }^{11}$ von Menschen verletzt werden, was schließlich das zivilcouragiertes Handeln eines Helfers auslöst (Meyer, 2004a, S. 27).

Die Definition von Zivilcourage enthält demnach folgende Merkmale:

öffentliches Handeln einer Person in einer bedrohlichen Situation,

zugunsten eines bedrohten oder geschädigten schwächeren Dritten,

- aufgrund gesellschaftlicher oder persönlicher Wertmaßstäbe bzw. Normen,

- mutig und durch Ärger begleitet,

in Interaktion mit einem Täter sowie

mit dem Risiko eines nicht vollständig abschätzbaren Eigenschadens.

Durch Oßwald, Frey, Greitemeyer \& Fischer wurde das ursprünglich von Latané \& Darley entwickelte Prozessmodell für Hilfeleistung um spezielle für Zivilcourage relevante Faktoren ergänzt. Einbezogen sind z. B. eine Kosten-Nutzen-Analyse, die Salienz gesamtgesellschaftlicher Normen sowie Empathie und Ärger als affektive Prozesse, die die Stimmungslage eines potenziellen

Abbildung 1: Prozessmodell für Zivilcourage

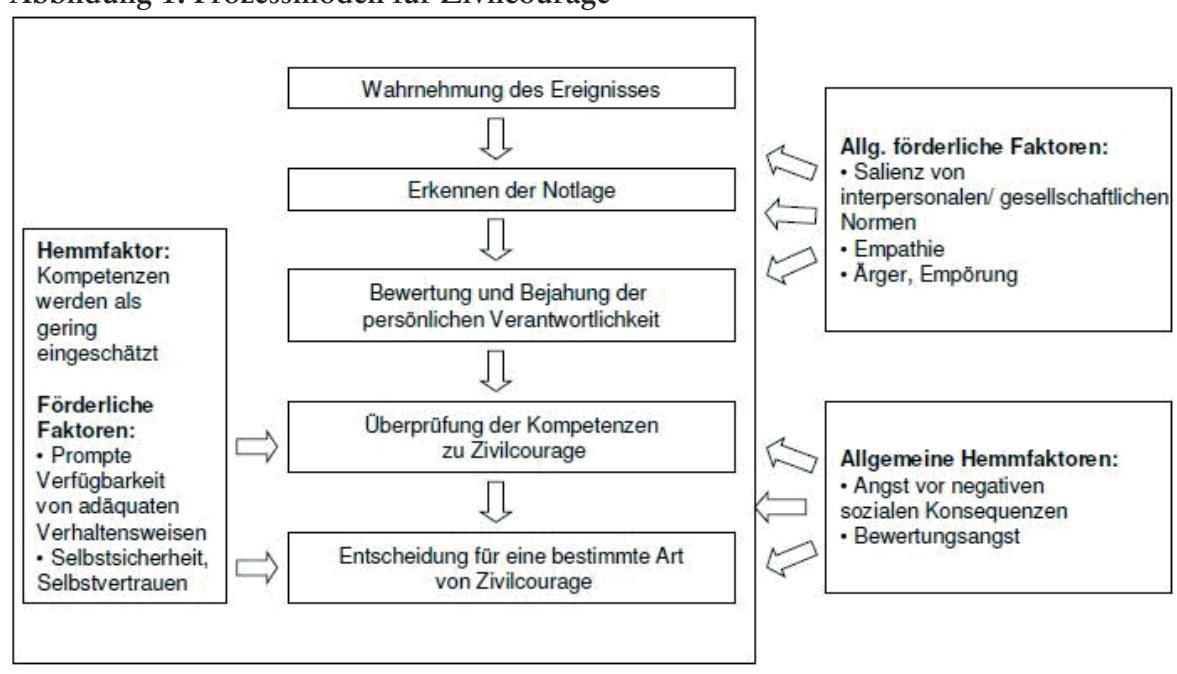

Quelle: Oßwald, Frey, Greitemeyer \& Fischer, 2007, S. 131

Die Untersuchung konzentriert sich ausschließlich auf akute Notsituationen ${ }^{9}$, die sofortiges Handeln erfordern. Zivilcourage kann aber auch bei länger andauernden Problemsituationen oder kritikwürdigen Umständen erforderlich sein. ${ }^{10}$ Die Situation, in der zivilcouragiert gehandelt wird, ist
Helfers mit einbeziehen (Oßwald et al., 2007, S. 123 ff.).

$\mathrm{Zu}$ den personenbezogenen Einflussfaktoren zählen - neben den körperlichen Eigenschaften des Helfers und einer eventuellen Bekanntheit mit dem Opfer - die 
sofortige Verfügbarkeit adäquater Verhaltensweisen für das Einschreiten (Jonas \& Brandstätter, 2004, S. 187 ff.) sowie die soziale Kompetenz des potenziellen Helfers, seine Fähigkeit zur schnellen Reaktion und die subjektive Einschätzung des couragierten Helfers zu seinem eigenen Artikulations- und Argumentationsvermögen (Meyer, 2004a, S. 141).

Der erwähnte „Bystander“-Effekt ist klassischer Fall einer situationsbedingten Determinante mit ggf. hemmender Wirkung bei auftretenden Effekten der „Verantwortungsdiffusion “ oder der „pluralistischen Ignoranz", bei der Helfer ihr persönliches Einschreiten als nicht notwendig erachten, da einer der anderen Anwesenden den Notfall beheben oder die Hilfe bereits unbemerkt eingeleitet haben könnte (Darley \& Latané, 1968, S. 377 f.). Bei offensichtlich hochgefährlichen Zivilcouragesituationen zeigt eine aktuelle Studie allerdings eine geringere Häufigkeit des „Bystander“-Effekts (Fischer et al, 2006, S. 275).

Entscheidungen zugunsten eines Einschreitens sind in hohem Maße sowohl von aktuellen gesellschaftlichen Wertmaßstäbe und Rechtsauffassungen als auch von sozialen Einflussfaktoren abhängig, wobei gezielten Appellen ein positiver Effekt auf ein Klima von Zivilcourage zugesprochen wird (Jonas \& Brandstätter, 2004, S. 187). Gegenteilige Auffassungen schreiben Werbekampagnen nur bedingt Einfluss auf prosoziales Verhalten zu, da extrinsische Motive für ein Eingreifen eine weniger ausgeprägte Rolle spielen (Bierhoff, 2004, S. 60 f.).

Offen bleibt die Frage, wie die Berichterstattung über tatsächliche Fälle von Zivilcourage wirkt und ob die regelmäßig enthaltenen Appelle zur "Nachahmung“ im positiven Sinne führen, oder - aufgrund der in einzelnen Fällen schlimmen Folgen für den Helfer bzw. nachträglicher Kritik an der Art und Weise des Eingreifens - ein hemmender Effekt eintritt? Das bloße Vorhandensein einer gesetzlichen Gebots zur Hilfeleistung ist jedenfalls nicht ausreichend, um annehmen zu können, dass diese immer umgesetzt wird. Vielmehr sind eine Übernahme der Norm mit deren Inhalten, sowie deren spezifische Aktivierung erforderlich (Oßwald et ${ }^{\circ}$ al, 2007, S. 125). ${ }^{12}$

„Zivilcourage wollen ist Eines, so handeln können ein Anderes, tatsächlich sozialen Mut beweisen ein Drittes. “(Meyer, 2004a, S. 127)

Die veröffentlichten Handlungsempfehlungen des Programms Polizeiliche Krimi- nalprävention der Länder und des Bundes (ProPK) in der Initiative "weggeschaut. ignoriert. gekniffen“ setzen Schwerpunkte bei der Aktivierung weiterer Personen als Unterstützung des Helfers und der Alarmierung der Polizei sowie auf die Vermeidung einer Eigengefährdung couragierter Bürger. Die Kampagne wirbt für „Hinsehen statt Wegschauen“ und „Engagement statt Gleichgültigkeit“ und empfiehlt „6 praktische Regeln für mehr Sicherheit im Alltag“ als Verhaltensweisen für ein Einschreiten in Zivilcouragesituationen (Internetseite des ProPK, „aktion-tu-was“).

Jonas und Brandstätter (2004, S. 192) stellen persönliche Eigenschaften wie Selbstsicherheit und die sofortige Verfügbarkeit adäquater Verhaltensweisen als bedeutende Faktoren für ein Einschreiten in einer Zivilcouragesituation dar. Durch wiederholtes Üben von Einschreitsituationen ist eine Steigerung der Kompetenz bei der Bewältigung der Standardverhalten (z. B. Durchführung des Notrufs, Erheben der Stimme) zu erreichen. Dies wiederum wirke sich positiv auf das Selbstvertrauen, Handlungsentschlossenheit und Handlungsroutine aus (ebda., S. 192). Nach Brandstätter müsse für „richtiges Handeln im richtigen Augenblick “ das gleichzeitige Vorliegen von Handlungswissen und Handlungskompetenz gegeben sein (2007, S. 270).

Obwohl zivilcouragiertes Handeln primär gewaltfreies Handeln darstellt (Meyer, 2004a, S. 15), kann körperliches Einschreiten etwa zur Verteidigung eines Dritten gegen eine Bedrohung oder einen körperlichen Angriff erforderlich sein (Nunner-Winkler, 2007, S. 30). Ohne im Detail auf rechtliche Aspekte und Rechtfertigungsgründe bei der Anwendung von Gewalt im Rahmen von Zivilcouragesituationen einzugehen, stellen das Beobachten und das korrekte Erkennen einer Notsituation und überdies die Anpassung des eigenen Verhaltens höchste Anforderungen an den potenziellen Helfer. Darüber hinaus ist fraglich, ob generell die Anforderungen hinsichtlich des Erkennens, Deutens und Beurteilens der Situation und der Handlungen aller Beteiligten vom Helfer entsprechend erfüllt werden können. Gleichwohl kann eine unterlassene Hilfeleistung einem Mitmenschen gegenüber unter bestimmten Voraussetzungen einen Straftatbestand erfüllen. ${ }^{13}$

\section{Datenbasis}

Datenmaterial der Studie sind 40 Lebenssachverhalte mit zivilcouragiertem Einschreiten, die Bestandteil polizeilicher Ermittlungsvorgänge sind und auf Ermittlungsakten und Vernehmungsprotokollen basieren. ${ }^{14}$ Durch die Analyse der Zivilcouragefälle sollen, vom empirischen Material ausgehend, typische Merkmale identifiziert werden, um anschließend mittels einer typologischen Analyse das Material strukturieren zu können (Mayring 2002, S. 132 ff). Die Auswahl und Filterung der Sachverhalte erfolgte gemäß der theoretischen Vorannahmen aus der bestehenden Hilfeleistungsund Zivilcourageforschung sowie der Erfahrungen aus der polizeilichen Praxis. So konnte sicher gestellt werden, dass nur die für die Untersuchungsfrage relevanten Sachverhalte einbezogen werden (Kelle \& Kluge, 2010, S. 43). Zielrichtung war einerseits die Begrenzung der Datenmenge auf ein adäquates Maß sowie andererseits „die Vermeidung von Verzerrungen bzw. der [ausschließliche] Einbezug von relevanten Fällen ...“ (ebda., S. 42). Im abschließenden Analyseschritt erfolgte der Vergleich der den Geschehensverlauf beeinflussenden Faktoren - sowohl innerhalb des Einzelfalles als auch über alle Zivilcouragefälle hinweg - wodurch Regelmäßigkeiten, Übereinstimmungen und Unterschiede erkennbar wurden.

\section{Deliktsbereiche}

Die den couragiertem Helferhandlungen zugrunde liegenden Straftaten sind den folgenden Deliktsbereichen zugeordnet, wobei - wegen der ggf. schwierigen Erkennbarkeit bzw. rechtlichen Unterscheidbarkeit aus Sicht eines Helfers - die versuchten Tötungsdelikte und Körperverletzungsdelikte zusammengefasst wurden:

Die meisten couragierten Helfer waren bei ihrem Eingreifen alleine bzw. lediglich zu zweit. In 25 Fällen stand ein Helfer einem Täter gegenüber, in weiteren acht Sachverhalten waren die Helfer zahlenmäßig überlegen.

\section{Situationselemente und Einschreitverhalten}

Die Fallanalysen der Zivilcourage-Fälle erforderten eine Betrachtung des gesamten Kontexts der jeweiligen Situation und beispielsweise nicht nur des Verhaltens des Helfers bei seinem Einschreiten. Die Handlungen von Täter und Opfer und die Umstände 
Abbildung 2: Verteilung der den Helferhandlungen zugrunde liegenden Deliktsbereiche

\section{Verteilung der Deliktsbereiche}

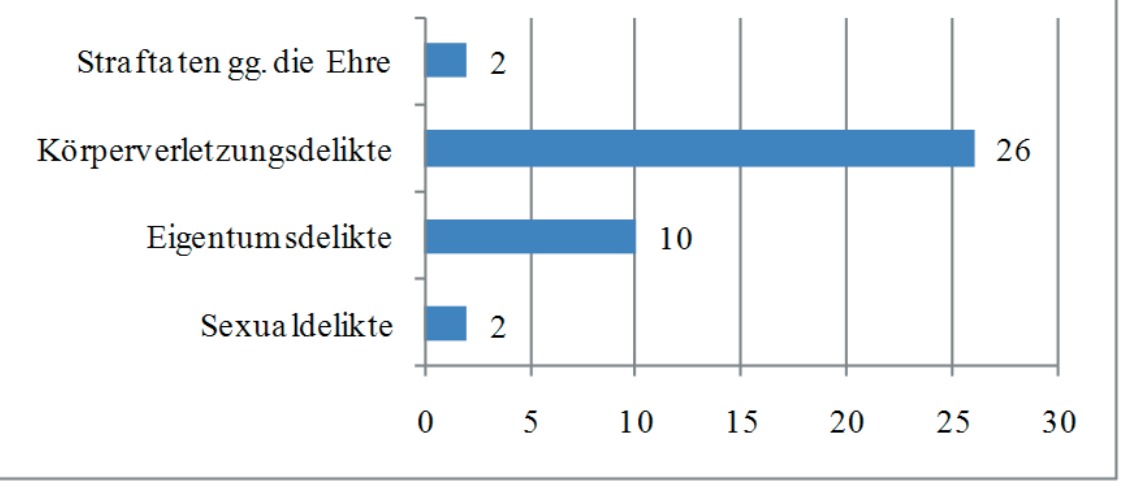

Abbildung 3: Anzahl von Helfern und Tätern innerhalb einzelner Fallkonstellationen und deren Häufigkeit bezogen auf alle Zivilcourage-Fälle

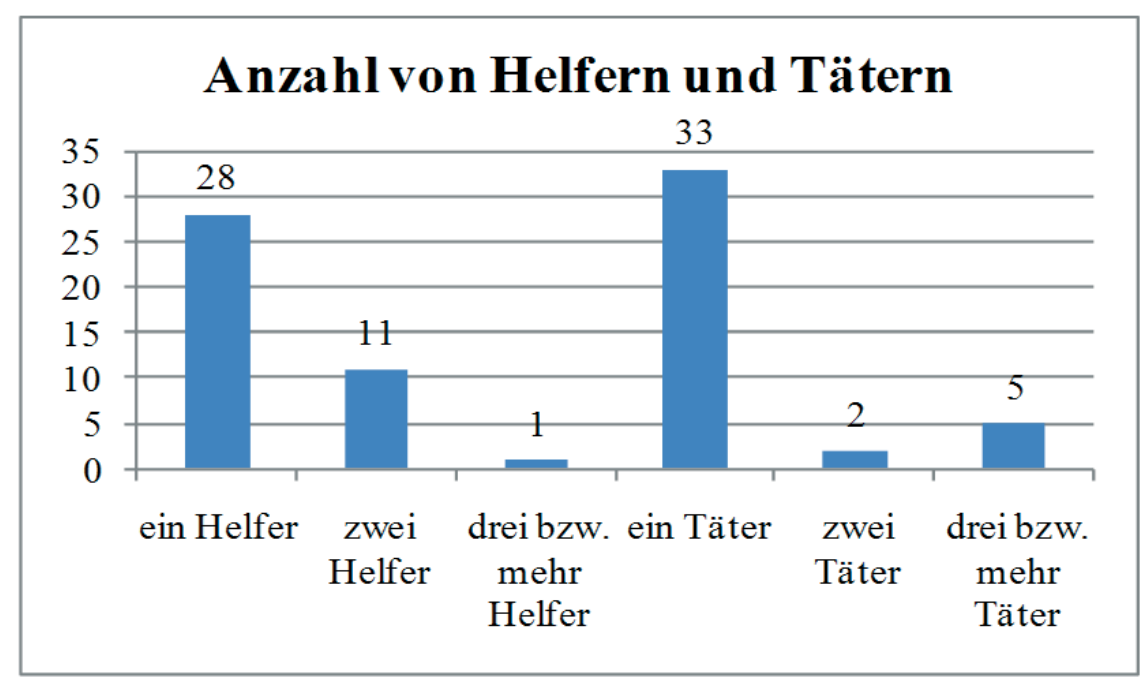

der Situation stellen ein Geflecht innerhalb eines isolierten sozialen Ereignisses dar, weswegen sie im Zusammenhang zu analysieren sind (Wolfgang, 1958; zitiert nach: Polk, 1994, S. 3).

Das konkrete Verhalten des Täters unmittelbar vor der Helferintervention ist bereits für die Zielrichtung bzw. Art und Weise seines Einschreitens relevant. Je nachdem, ob die Tathandlung noch andauert oder ob der Täter diese bereits beendet hat und sich vom Tatort entfernen will, werden unterschiedliche Ziele verfolgt:

eine Hilfeleistung zugunsten des Opfers (Verhinderung einer drohenden Tat, Unterbindung der Tathandlung), bzw.

das Festhalten des Täters, entweder nach erfolgreicher Intervention oder auch ausschließlich, ggf. im Anschluss an eine Verfolgung.
Das Einschreiten eines couragierten Bürgers bezieht sich, trotz des vielfach benutzten Terminus „Hilfeleistung“, in allen untersuchten Fällen auf eine Interaktion mit dem Täter, durch die die Tathandlungen beendet bzw. die Festhaltung des Täters erreicht werden sollte. Den Opfern wurde insoweit nur mittelbar geholfen. Wenngleich sich die Handlungsmöglichkeiten eines Helfers innerhalb der Interaktion sehr unterschiedlich gestalten können, haben die couragierten Bürger tatsächlich nur wenige Möglichkeiten genutzt. Unabhängig von der Entfernung zum Täter besteht für den Helfer meist die Möglichkeit, verbal mit diesem zu interagieren, wobei durch Zurufe gleichzeitig auch die Aufmerksamkeit weiterer Personen erregt werden kann (Nunner-Winkler, 2007, S. 30). Den Umständen entsprechend kann dem Helfer ein (rein) verbales Eingreifen zur Bereinigung der Notsituation nicht Erfolg versprechend erscheinen, sodass er zur Ab- wehr einer weiteren Schädigung des Opfers ein körperliches Einschreiten für erforderlich hält (ebda., S. 30).

Die Ausprägung des Merkmals „Einschreitverhalten“ erfolgte für eine weitere Analyse nach den Dimensionen:

„verbal“,

- „erfolglos verbal und initiativ körperlich“ (z. B. bei Fortführung der Tathandlung) sowie

„unmittelbar initiativ körperliches Einschreiten“ (z. B. bei hoher Dringlichkeit der Intervention).

Mit „initiativ körperlichem Einschreiten“ ist das bewusste und zielgerichtete körperliche Eingreifen des Helfers gemeint, ohne vorheriges verbales Ansprechen bzw. ohne einen vorausgegangenen Angriff bzw. ohne Bedrohung des Helfers.

Wie die untersuchten Sachverhalte zeigen, fand im Regelfall die Helferintervention ohne eine vorherige ausgeprägte Situations-/ Risikoanalyse des Helfers statt, wobei generell die folgende Überlegungen bei einer Risikoanalyse möglich sind. Zunächst erscheint plausibel, dass der Helfer bei der Einschätzung der Situation, seinem eigenen Interesse folgend, die ihm am ungefährlichsten und zugleich am ehesten Erfolg versprechend erscheinende Handlungsoption wählt (Meyer, 2004a, S. 264). Je größer sich die Wahrscheinlichkeit des Erfolgs bei der Klärung der kritischen Notsituation darstellt, desto eher kann ein Eingreifen angenommen werden. Ebenso nimmt die vermutete Eigenschadenshöhe Einfluss, wobei mit zunehmendem Eigenrisiko die Bereitschaft zum Handeln eher zurückgehen wird (Piliavin \& Piliavin, 1972; zitiert nach: Bierhoff, 2006, S. 120 f). Hintergrund dieser Überlegungen ist die Frage, ob ein Helfer einen erheblichen Eigenschaden „bewusst“ in Kauf nehmen würde, wenn nur geringe Normverletzungen wie Beleidigungen oder einfache Körperverletzungen vorliegen, die nicht direkt auf einen zu erwartenden schweren Fremdschaden hinweisen. Die häufigste Variante müsste demnach ein Eingreifen im Falle sowohl eines geringen Misserfolgs-Risikos als auch mit geringer Erwartung an einen möglichen Eigenschaden darstellen. Bei einem möglichen hohen Eigenschaden muss - unabhängig vom Risiko des Misserfolgs der Intervention an sich - eine besonders hohe Dringlichkeit der Hilfeleistung vorliegen bzw. Helfer müssen den Eintritt eines Schadens für unwahrscheinlich halten. An- 
dernfalls käme dieses „fast aussichtslose“ Handeln einem „Aufopfern“ gleich.

Eine umfassende Einschätzung der Gefährlichkeit der Situation ist aber für den Helfer denkbar schwierig und ggf. fehlerbehaftet. Die Gefahreneinschätzung ist ihm sowohl nur anhand der aktuellen und für ihn wahrnehmbaren Situation möglich und leidet darüber hinaus an der Unvorhersehbarkeit und Unberechenbarkeit zwischenmenschlicher Interaktionen (Füllgrabe, 1999, S. 31). Eine „subjektive Handlungssicherheit“ (Meyer, 2004a, S. 45) kann beim couragierten Bürger zur Fehleinschätzung von spezifischen Gefahren (Verhalten des Täters, situative Umstände) sowie seiner eigenen Fähigkeiten führen. Obwohl anzunehmen ist, dass Helfer im Rahmen einer Befragung angeben würden entsprechende Maßnahmen zur Risikobegrenzung durchgeführt zu haben, kann die Analyse Geschehensverläufe dies nur in drei Fällen - hier jeweils durch die bewusste Wahl eines Sicherheitsabstandes belegen.

\section{Körperliche Auseinandersetzungen}

Die körperlichen Auseinandersetzungen in den untersuchten Sachverhalten entsprechen zumeist dem Muster sog. „one-punchaffairs", also Kämpfen in denen derjenige „gewinnt", welcher den ersten Schlag führt (Collins, 2008, S. 15). Reale Gewalt entspricht hier nicht den fiktiven (Maurer \& Reinemann, 2006, S. 168; ebenso Kersten, 2008, S. 293) Darstellungen in Film und Fernsehen, die lang andauernde Kämpfe nahezu gleichstarker Gegner zeigen (Collins, 2008, S. 14 f). Vielmehr sind in der Realität länger andauernde verbale Auseinandersetzungen und nur kurze Sequenzen mit tatsächlicher Gewaltausübung zu beobachten. Die Dauer der körperlichen Auseinandersetzungen kann in den untersuchten Fällen nicht detailliert bestimmt werden, allerdings überwiegen solche kürzer als eine Minute, da entweder ein Beteiligter bereits nach kurzer Zeit aufgab oder derart verletzt war, dass nicht weiter auf ihn eingeschlagen wurde.

\section{Schadensart und -höhe}

Hinsichtlich des erlittenen Schadens der beteiligten Opfer/Geschädigten war für eine weitergehende Analyse die Bestimmung zusätzlicher Ausprägungen erforderlich. Die nachfolgenden Unterkategorien beziehen neben einem tatsächlich eingetretenen Schaden auch den Aspekt der vom Beobachter/
Helfer wahrgenommenen Gefährlichkeit mit ein:

- geringer Schaden (z. B. Drohungen, Ohrfeigen, bloßes Schubsen),

- mittlerer Schaden (z. B. Faustschläge auf Körper und Gesicht, Umwerfen/Niederschlagen des Opfers) sowie

- hoher Schaden (sichtbare Blutungen, Schläge/Fußtritte gegen Körper und Kopf eines auf dem Boden liegenden Opfers, Zuschlagen mit Gegenständen). ${ }^{15}$

Der Kategorisierung folgend erlitten vier Opfer mittleren bis hohen Schaden, 20 Opfer mittleren Schaden und bei zehn Opfern war lediglich geringer Schaden festzustellen bzw. noch kein Schaden eingetreten. ${ }^{16}$

\section{Typologie von Zivilcourageverhalten}

Die Konstruktion der Falltypen erfolgte über die zwei am bedeutendsten erscheinenden Einflussfaktoren auf den gesamten Geschehensverlauf, nämlich die grundlegende Zielrichtung des Einschreitens der couragierten Helfer sowie die konkrete Art und Weise des Helferverhaltens. Dabei wurden die Fälle, die hinsichtlich ihrer Merkmale Regelmäßigkeiten zeigen, zu einem Typ gruppiert (interne Homogenität), wodurch sich zugleich Unterschiede zu anderen Gruppen (externe Heterogenität) ergaben (Kelle \& Kluge, 2010, S. 93). Als Vergleichsdimensionen wurde das objektive Helferverhalten beim Einschreiten herangezogen, unterscheidbar nach:

\section{- ausschließlich verbalem Einschreiten; \\ zunächst verbalem, dann körperlichem Einschreiten; \\ - unmittelbar körperlichem Einschreiten.}

Als zweite Dimension wurde die Zielrichtung des Einschreitens zur Typenbildung beigezogen:

- Einschreiten als Hilfeleistung zur Verhinderung eines Schadenseintritts bzw. einer Schadensausweitung;

Einschreiten zur Festhaltung des flüchtenden Täters.

\section{„Dominierende Zivilcourage“}

Prägend für den Falltyp der „dominierenden Zivilcourage " ist ein Einschreitverhalten des Helfers, bei dem dieser den Geschehensverlauf nahezu alleine bestimmt. Die Art und Weise „dominierender“ Helferhandlungen lassen sich zwar in Teilen in der tatsäch- lichen Durchführung unterscheiden, im Kern verläuft die Interaktion aber zielorientiert und im angestrebten Handlungsrahmen des Helfers. Zwei grundlegende Helferverhaltensweisen lassen sich diesem Falltyp bestimmen:

a) Das Einschreiten des Helfers „überwältigt" den Täter unmittelbar und beraubt ihn dabei seiner Einflussmöglichkeiten. Gleichzeitig wird durch das Einschreiten des Helfers auch die ursprüngliche Tathandlung unterbunden, sodass keine weiterer Schaden beim Opfer eintreten kann. Von den 16 Einschreitverhalten sind couragierte Helfer achtmal - initiativ körperlich - gegen den Täter vorgegangen und haben den Vorteil des Überraschungsmoments (zumindest unbewusst) genutzt.

b) Bei der zweiten Variante ordnet sich der Täter dem dominierenden Verhalten des couragierten Bürger unter und gibt seine Tathandlung „freiwillig“ auf. Dies ist in Fällen dokumentiert, bei denen der Täter den Vorgaben des Helfers ohne Anwendung körperlicher Gewalt, nur aufgrund direkten Ansprechens durch den Helfer, gefolgt ist.

Die bedeutenden Merkmale der „dominierenden Zivilcourage“ sind:

Tathandlung steht unmittelbar bevor (Gefährdung) oder wird bereits ausgeführt (Schädigung);

Zielrichtung des Einschreitens ist die Verhinderung einer Schädigung/Schadensausweitung beim Opfer;

schnelles Eingreifen des Helfers ohne lange Planungsphase, damit keine oder minimale Risikoabwägung hinsichtlich einer Eigengefährdung;

hohe Dringlichkeit der Hilfeleistung wegen bereits eingetretener Schäden oder des zu erwartenden Schadensausmaßes (im Durchschnitt höher als bei „zögerlicher Zivilcourage");

\section{hoher Erfolgswille.}

Die Auswertung der zugehörigen Sachverhalte zeigte in allen Fällen ein außerordentlich schnelles und konsequentes Eingreifen. Bemerkenswert ist, dass lediglich in zwei Fällen innerhalb dieser Typengruppe ein Notruf vor dem Einschreiten durchgeführt wurde, jeweils im Auftrag des direkt gegen den Täter einschreitenden Helfers. Als Grund für das schnelle Eingreifen in Anbetracht der jeweiligen Situation ist denkbar, 
dass der Eindruck der Dringlichkeit der Hilfeleistung für den Helfer eine besondere Rolle spielt, wenn z. B. bereits eindeutige Verletzungszeichen sichtbar sind oder die Tathandlung ein hohes Schädigungspotenzial erwarten lässt. ${ }^{17}$ Zum anderen ist es dem Helfer durch das Überwältigen des Täters möglich, das Eigenrisiko zu minimieren und einer Gewaltausübung des Helfers zuvorzukommen. Das Helferverhalten erscheint als ein spontanes und impulsives Handeln, ohne jegliche Abwägung des Risikos (Piliavin \& Piliavin, 1972; zitiert nach: Bierhoff, 2006, S. 120 ff).

Während sich zwei der insgesamt 16 Helfer bei ihrem Einschreiten mehr als nur geringfügig verletzt haben, blieben 14 Helfer unverletzt bzw. waren etwaige Verletzungen nicht dokumentiert. Weitere negative Kosten, z. B. beschädigte oder verschmutzte Kleidungsstücke, blieben unberücksichtigt.

\section{„Zögerliche Zivilcourage“}

Der „zögerlichen Zivilcourage“ sind acht Sachverhalte zugeordnet. Charakteristisch ist ein verbales Einschreiten, welches den Täter dazu bewegen soll, sein normverletzendes Handeln (selbst) zu beenden.

Das Eingreifen der couragierten Helfer begann in allen Fällen mit einer verbalen Kommunikation, in deren Verlauf der Täter zum Unterlassen einer drohenden bzw. zur Beendigung einer bereits schädigenden Handlung aufgefordert wurde. In zwei Fällen erfolgte neben dem Ansprechen, mit Formulierungen wie: „Das geht nicht“, „Lassen Sie das“, „leichtes“ körperliches Anfassen durch leichtes Wegziehen oder Wegschieben des Täters vom Opfer. Die eher zögernde Vorgehensweise, gegen die sich der Täter ohne oder nur mit geringem Aufwand hätte durchsetzen können, gab dabei den Anstoß für die Bezeichnung des Falltyps.

Die Intervention der ,zögerlichen Zivilcourage“ zielt darauf ab, primär die Aufmerksamkeit des Täters zu erreichen und sie vom Opfer wegzulenken, um danach, im Rahmen einer weiteren verbalen Interaktion, eine endgültige Beendigung der Tathandlung herbeizuführen.

Die „zögerliche Zivilcourage“ zeichnet sich dabei ebenfalls durch spontane Handlungen des Helfers aus, weswegen hier ebenso nicht von einem gezielten Abwägen unterschiedlicher Einschreitmöglichkeiten und einer bewussten Auswahl der risikoärmsten Variante auszugehen ist.
Die kennzeichnenden Merkmale der „zögerlichen Zivilcourage " sind:

- Tathandlung steht unmittelbar bevor (Gefährdung) oder wird bereits ausgeführt (Schädigung);

Zielrichtung des Einschreitens ist die Verhinderung einer Schädigung/Schadensausweitung beim Opfer

- Eingreifen des Helfers ohne lange Planungsphase, damit keine oder nur minimale anzunehmende Risikoabwägung hinsichtlich einer Eigengefährdung;

hohe Dringlichkeit der Hilfeleistung wegen bereits eingetretener Schäden oder des zu erwartenden Schadensausmaßes (Schäden im Durchschnitt eher geringer als bei der „dominierenden Zivilcoura$\mathrm{ge}^{\text {") }}$;

Das im Vergleich zur „dominierenden Zivilcourage " weniger energische Helferhandeln grenzte den Handlungsspielraum der Täter nicht ein und bot z. B. durch Betreten des unmittelbaren Nahbereichs des Täters durch den Helfer zugleich zusätzliche Ansätze für einen unerwünschten Verlauf. Generell können Täter solche, zögernd durchgeführte Interventionen als Anzeichen fehlender Handlungskompetenz bzw. als nur gering ausgeprägten Helferwillen in Bezug auf eine drohende Konfrontation oder eine mögliche Verfolgung deuten. Mit anderen Worten kann für den Täter der Eindruck entstehen, in der Person des Helfers einen „schwachen " Gegner vor sich zu haben, wobei das „zögerliche“ Einschreiten - als Indiz für fehlende Selbstsicherheit - die Wahrscheinlichkeit, dass der Helfer selbst zum Opfer wird, erhöht (Füllgrabe, 2003, S. 154). Dies zeigt sich insoweit auch bei der Auswertung der von den Helfern erlittenen Schäden, da von acht Helfern fünf zumindest geringen Schaden erlitten und in einem Falle ein Helferschaden unmittelbar bevorstand, aber durch das Eingreifen eines Dritten verhindert wurde. Die Vorgehensweise der Helfer vermittelt den Eindruck, dass zwar entsprechender Wille zum Einschreiten vorhanden war, aber die Helfer aufgrund mangelnder Erfahrung, fehlendem Selbstvertrauen, Wissen oder Training, dies - im Sinne der dargestellten Handlungsempfehlungen - nicht anders dazu in der Lage waren. Obwohl die Notsituationen im Vergleich zu denen der dominierenden Zivilcourage eine eher geringere Dringlichkeit eines sofortigen Handelns aufwiesen, wurde in keinem der Fälle vor dem Einschreiten ein Notruf abgesetzt.

\section{„Eskalierende Zivilcourage“}

Ein zweiphasiger Geschehensverlauf ist das wesentliche Merkmal der „eskalierenden Zivilcourage“, bei der ein Helfer - wie bei den bisherigen Interventionen auch - mit dem Bedroher bzw. Täter in Interaktion tritt, um einem Opfer Hilfe zu leisten. Im weiteren Verlauf des couragierten Einschreitens ist ein Zeitpunkt identifizierbar, ab dem der Helfer aufgrund bestimmter Umstände nicht mehr der ursprünglichen Zielrichtung der Hilfeleistung folgend handelt. Der Fokus des Helfers wendet sich vielmehr einem neuen Motiv zu, z. B. einer verbalen - persönlichen - Auseinandersetzung mit dem Täter. Ab diesem Zeitpunkt steht die Inkaufnahme negativer Kosten nicht mehr in direkter Verbindung zur ursprünglich beabsichtigten Hilfeleistung. Für die Helfer ergab sich mit der neuen Zielrichtung des Handelns und dem geänderten Geschehensverlauf einen besonders hoher Gefährdungsgrad, da sie selbst direkt Zielobjekt des Angriffs des Täters wurden. Grundsätzlich kann sowohl von Seiten des Täters wie von Seiten des Helfers die Spirale der Eskalation ausgelöst werden, z. B. durch Beleidigungen oder direkte Provokationen. Unerheblich ist, ob dies bewusst oder unbewusst geschieht, letztlich ist nur entscheidend, wie das Gegenüber reagiert. Die sich weiterentwickelnde Spirale der Eskalation beruht auf einem Aktions-/Reaktionsautomatismus, im Rahmen des gegenseitigen Aufschaukelns wird nur noch auf das Verhalten des jeweils anderen reagiert (Lünse, 2006, S. 48). Eng an der Untersuchungsfrage orientiert sind bei diesem Falltyp, der bei den 40 untersuchten Einschreitsituationen insgesamt drei Mal festzustellen war, die situativen Umstände und bewussten bzw. unbewussten Verhaltensweisen des Helfers von Interesse, die für den veränderten Verlauf verantwortlich sind. Die Umstände die zum „eskalierenden "Verlauf führten, waren in allen drei Fällen in erster Linie durch Inhalte der vorausgegangenen verbalen Interaktion beeinflusst. Es handelte es sich einmal um eine Provokation, die vom Helfer ausgesprochen wurde, sowie eine Provokation des Täters auf die der Helfer eingegangen war. Im dritten Fall kündigte der Helfer einen Notruf an, wodurch auch die eigentliche Tathandlung seitens der Tätergruppe beendet wurde. Durch den implizierten Hinweis, dass die Alarmierung der Polizei noch nicht stattgefunden hatte, geriet der Helfer selbst ins Visier der Täter. Die Drohung, die Polizei zu rufen, wurde damit „beantwortet“, dass ein Täter das Mobiltelefon des Helfers beschä- 
digte, worauf der Helfer eine körperliche Auseinandersetzung begann. Durch den sich hier den Tätern bietenden Handlungsspielraum - sie waren in der Überzahl und nicht gezwungen sofort zu flüchten - konnten sie ihre Aufmerksamkeit dem Helfer zuwenden. Der Falltyp der „eskalierenden Zivilcourage“ zeichnet sich durch folgende Merkmale aus:

- Tathandlung steht unmittelbar bevor (Gefährdung) oder wird bereits ausgeführt (Schädigung);

- Zielrichtung des Einschreitens ist die Verhinderung einer Schädigung/ Schadensausweitung beim Opfer - ggf. begleitet durch Vorliegen eines zweiten Motivs;

- eher niedrige Dringlichkeit der Hilfeleistung in Bezug auf die bereits erkennbaren oder noch zu erwartenden Schäden (im Durchschnitt geringer als bei „zögerlicher Zivilcourage“);

verbale Interaktion steht im Vordergrund; als Diskussion/Streitgespräch;

- Provokationen werden Bestandteil der verbalen Interaktion des Helfers mit dem Täter bzw. werden vom Helfer angenommen und erwidert;

Entwicklung einer separaten Handlungssequenz mit einer direkten Auseinandersetzung zwischen Helfer und Täter.

Insbesondere beim Falltyp der „eskalierenden Zivilcourage“ wird deutlich, dass der Geschehensverlauf - neben den Umständen der Situation - eng mit den Handlungen sowohl des Täters als vor allem auch des couragierten Helfers verflochten sind. Provokationen des Helfers stellen im Sinn der „Victim Precipitation Theory"18 von Wolfgang, Handlungen dar, die einen maßgeblichen Anteil am Verlauf der Situation und dem selbst zum Opfer werdenden Helfers haben kann (Wolfgang, 1970, S. 569 f.)

\section{„Exekutive Zivilcourage“}

Der Falltyp der „exekutiven Zivilcourage“ stellt innerhalb der konstruierten Typen einen Spezialfall dar, da neben Auffälligkeiten beim zugrunde liegenden Deliktsbereich sich insbesondere in Bezug auf die Zielrichtung und Motivation für das Einschreiten zusätzliche Aspekte ergeben haben. Typisch für Fälle der „exekutiven Zivilcourage“ ist, dass der Helfer über die unmittelbare Hilfeleistung zugunsten des Opfers hinaus bzw. ausschließlich im Anschluss an eine beende- te Tathandlung aktiv wird. Die unter diesen Falltyp subsumierten Sachverhalte beziehen sich nämlich auf die Festhaltung bzw. Verfolgung eines flüchtenden Täters durch einen Helfer. Eine „exekutive Zivilcourage “ war in 17 der 40 Helferverhaltensweisen festzustellen, in vier Fällen erfolgte die Verfolgung des Täters im Anschluss an eine vorherige Hilfeleistung und Unterbindung/ Abwehr der Tathandlung. In dreizehn Sachverhalten war dieses spezielle Einschreitverhalten einzig im Anschluss an eine bereits beendete Tathandlung erfolgt. so z. B. Raubstraftaten, bei denen typischerweise die unmittelbare Tathandlung noch vor einer etwaigen Helferintervention beendet ist.

Die bedeutenden Merkmale der ,exekutiven Zivilcourage“" sind:

die ursprüngliche Tathandlung ist in allen Fällen beendet;

geringe Dringlichkeit; das Einschreiten hat keinen/kaum Einfluss auf das Schadensausmaß;

- schnelles Eingreifen des Helfers ohne lange Planungsphase, damit auch keine oder lediglich minimale Risikoabwägung einer Eigengefährdung;

- Zielrichtung des Einschreitens ist "die Ergreifung des Täters“ - im Anschluss an eine vorherige Intervention zur Beendigung einer Tathandlung oder nur daran anschließend;

\section{- hoher Erfolgswille.}

In zehn der dreizehn Sachverhalte hatten die Helfer die Tathandlung nicht unmittelbar selbst gesehen, sondern wurden durch Hilferufe der Opfer oder anderer Tatzeugen aufmerksam. Dennoch beteiligten sie sich an einer Verfolgung bzw. führten diese sogar auch alleine weiter, sogar ohne selbst Zeuge der Ursprungstat gewesen zu sein bzw. Detailkenntnisse zur Schwere der Tat und zum Tatort zu haben. Zur Ablauf der Tathandlung sowie zur eventuell angewendeten Gewalt bzw. Bewaffnung des Täters war diesen Helfer ebenfalls nichts bekannt.

Bezogen auf die Zielrichtung des Helfers bei einer drohenden oder bereits andauernden Schädigung des Opfers, z. B. einer Körperverletzung, stellt das Einschreiten der „exekutiven Zivilcourage“ nicht mehr primär eine Hilfeleistung im engeren Sinn dar. Abhängig von der Ursprungstat kommen vielmehr die Reduzierung des finanziellen Schadens beim Opfer sowie die Feststellung der
Identität des Täters für etwaige zivilrechtliche Ansprüche in Betracht; vor allem aber auch die Sicherstellung der Strafverfolgung, also der Wille, den Täter einer Bestrafung zuzuführen, beispielsweise wenn der Helfer trotz bereits „gesicherter Beute“ die Verfolgung weiter durchführt. Denkbar ist auch, dass die in der Hilfeleistungsforschung untersuchten positiven Kosten in Form von Anerkennung, z. B. der Dank des Opfers oder umstehender Zeugen sowie eine zu erwartende Darstellung in den Medien für den Helfer eine Rolle bei der Entscheidung zum Einschreiten spielen. Bei alleiniger Betrachtung der äußerlichen Umstände drängt sich - unabhängig von der tatsächlichen Motivation - das Bild einer "Jagd“ auf den flüchtenden Täter auf. Welche Überlegungen im Moment der Entscheidung tatsächlich den Ausschlag gaben, bleibt unklar, entsprechende Aussagen sind nicht Inhalt der polizeilichen Vernehmungen. Die Gefahren einer Verfolgung liegen insbesondere auch in der Situation einer sich aussichtslos gestaltenden Flucht, die beim flüchtenden Täter den Entschluss zum Kampf, bedingt durch den physiologischen Prozess der sog. ,fightor-flight"-Reaktion ergeben kann (Zimbardo \& Gerrig, 2008, S. 469 ff; ebenso Friedman, 2010, S. 66). Fraglich ist ohnehin, ob ein „durchschnittlicher“ Helfer beim Entschluss zur Verfolgung bereits Vorstellungen vom weiteren Verlauf hat, vor allem in Bezug auf seine Handlungsmöglichkeiten am Ende der Verfolgung. Strebt er an, den Täter zu stellen und ihn ggf. mithilfe einer körperlichen Auseinandersetzung zu überwältigen? Oder soll die Verfolgung so lange dauern, bis entweder der Täter körperlich nicht mehr in der Lage ist, die Flucht fortzusetzen oder der Helfer an Informationen kommt, die eine nachträgliche (zweifelsfreie) Ermittlung zulassen.

Auch bezogen auf den Erfolgswillen zeichnen sich die Fälle der „,exekutiven Zivilcourage" besonders aus: Solange die Helfer keinen echten Schaden erlitten, blieben selbst Drohungen mit Schlagwerkzeugen - und in einem Fall auch mit einer Schusswaffe gegen die Helfer erfolglos und hielten diese nicht von ihrem Vorhaben ab. In drei der 17 untersuchten Sachverhalte der „exekutiven Zivilcourage“ haben Helfer Verletzungen durch den flüchtenden Täter erlitten. Nur zwei Täter konnten durch ihre Angriffe letztlich entkommen, alle anderen Verfolgungen verliefen für die Helfer erfolgreich.

Aufgrund der absolut dringlichen Entscheidung zum Eingreifen durch die drohende 
Flucht, ist bei diesem Falltyp ein hoher Anteil an spontanem, impulsivem Helferverhalten gegeben. Eine Risikoabwägung, die über das Maß einer oberflächlichen Augenblickseinschätzung hinaus geht, ist deswegen anzuzweifeln. In jedem Fall stellt eine Verfolgung eine Situation mit hohem Gefahrenpotenzial für den Helfer dar, die im Kern allen propagierten Verhaltensweisen für „gefahrloses Einschreiten “ widerspricht (Internetseite des ProPK, ,Aktion-tu-was“).

\section{Untersuchungsergebnisse}

Besonders auffallend und darüber hinaus in nahezu allen Sachverhalten gleich, ist der außerordentlich kurze Zeitraum zwischen der Wahrnehmung der Nothilfe-Situation bis zum Entschluss und dem tatsächlichen Einschreiten des couragierten Bürgers. Die Kürze des Zeitraums legt weniger ein „bewusstes" Durchdenken der beschriebenen Entscheidungsstufen in den beschriebenen Prozessmodellen als vielmehr reflexartiges Helferhandeln nahe. Die Entscheidung zum Einschreiten in Notlagen bzw. Zivilcouragesituationen scheint bereits vor einem Ereignis getroffen, entweder durch die Sozialisation des Betroffenen oder eine bewusste Auseinandersetzung mit der Thematik. Der in den Fällen der „dominierenden“ und „zögerlichen Zivilcourage“ darlegte hohe Handlungsdruck ist geeignet, in hochgefährlichen Notsituationen als ergänzender Faktor intuitives Einschreiten zu forcieren (Meyer, 2004a, S. 34).

Eine schnelle Reaktion ohne Risikobewertung der Situation bedeutet grundsätzlich auch die - zumindest unbewusste - Inkaufnahme eines erhöhten Risikos. Dazu zählt auch die „Wahl“ der eigenen Vorgehensweise, die mehr von der Dringlichkeit der Situation und der Persönlichkeit des Helfers beeinflusst ist, als dass sie einer gezielten Entscheidung unterliegt (ebda., S. 45).

Die Untersuchungsergebnisse lassen Ansätze auf risikoreiche und risikoärmere Verhaltensweisen couragierter Helfer erkennen. Beispielsweise wurde die Verständigung der Polizei und die Hinzuziehung weiterer Helfer als wünschenswerte Standardmaßnahme kaum verwirklicht. Darüber hinaus stellen die Verfolgung eines flüchtenden Täters bei der „exekutiven Zivilcourage“ und der Beginn einer verbalen Intervention erst nach Erreichen des unmittelbaren Nahbereichs des Täters beim Falltyp der „zögerlichen Zivilcourage" weitere bedeutende gefahrenerhöhende Momente dar. Wenngleich die geringe Fallzahl keine repräsentative Aussage zulässt, so wurden doch in den acht Sachverhalten dieses Falltyps fünf ,zögerliche" Helfer verletzt. Dem gegenüber erhöht offenbar die Bestimmtheit des Eingreifens und damit einhergehende Eingrenzung des Handlungsspielraums des Täters, analog der „dominierenden Zivilcourage“, die Wahrscheinlichkeit eines positiven Verlaufs.

Auch wenn in dieser Untersuchung akute Notlagen, mit teilweise problematischen Geschehensverläufen und auch Helferschaden im Fokus standen, ist anzunehmen, dass das Gros der „alltäglichen Normalfälle“ bei Zivilcourage-Situationen gewaltfrei abläuft (Meyer, 2004a, S. 15). Gefahren für den Helfer bleiben dennoch jedem Fall inhärent und entsprechend themenbezogene Präventionsarbeit scheint erforderlich. So z. B. in Bezug auf die bisher wenig erfolgreichen Appelle, im Notfall die Polizei zu verständigen und weiterer Zeugen beizuziehen. Deutlich zeigt sich die Notwendigkeit der Vermittlung von Handlungskompetenz, ohne die eine adäquate Umsetzung theoretisch richtiger Verhaltensweisen zur Lösung von Zivilcouragesituationen nicht möglich ist (Frey, Brandstätter, Peus \& Winkler, 2004, S. 2). Zu hoffen bleibt, dass sich Vorfälle mit schweren oder den schwersten denkbaren Folgen für den Helfer, wie im Fall „Dominik Brunner", nicht wiederholen.

\section{Literaturverzeichnis}

Baringhorst, S. (2001). Solidarität - Erlebnisaspekt und Selbstinszenierung. In H.-W. Bierhoff \& D. Fetchenhauer (Hrsg.), Solidarität. Konflikt, Umwelt und Dritte Welt (S. 253272). Opladen: Leske und Budrich.

Batson, C. (1998). Altruism and prosocial behaviour. In D. Gilbert, S. Fiske \& G. Lindzey (Hrsg.), Handbook of social psychology (4. Auflage, Band 2, S. 282-316). New York: Oxford University Press.

Bayerisches Datenschutzgesetz in der Fassung der Bekanntmachung vom 23. Juli 1993 (GVBL S. 498, BayRS 204-1-I), zuletzt geändert durch $\$ 1$ des Gesetzes vom 27. Juli 2009 (GVBL S. 400).

Bierhoff, H.-W. (2004). Handlungsmodelle für die Analyse von Zivilcourage. In G. Meyer, U. Dovermann, S. Frech \& G. Gugel (Hrsg.): Zivilcourage lernen: Analysen - Modelle Arbeitshilfen (S. 60-69). Bundeszentrale für politische Bildung, Landeszentrale für politische Bildung Baden-Württemberg.

Bierhoff, H.-W. (2006). Sozialpsychologie: Ein Lehrbuch (6. überarbeitete und erweiterte Auflage). Stuttgart: Kohlhammer.

Bierhoff, H.-W. (2010). Psychologie prosozialen Verhaltens: Warum wir anderen helfen (2., vollständig überarbeitete Auflage). Stuttgart: Kohlhammer.

Brix, E., Nautz, J. \& Thien, K. (Hrsg.) (2006). Zivilcourage. Wien: Passagen Verlag.

Collins, R. (2008). Violence: A Micro-sociological Theory. Princeton: Princeton University Press.

Darley, J. \& Latané, B. (1968). Bystander intervention in emergencies: Diffusion of respon- sibility. Journal of personality and Social Psychology, 8(4), 377-383.

Fischer, P., Greitemeyer, T., Pollozek, F. \& Frey, D. (2006). The unresponsive bystander: Are bystanders more responsive in dangerous emergencies? European Journal of Social Psychology, 36, 267-278.

Frey, D., Brandstätter, V., Peus, C. \& Winkler, M. (2004). Zivilcourage: Intoleranz gegenüber Intoleranz. In H. R. Yousefi \& K. Fischer (Hrsg.), Interkulturelle Orientierung - Grundlegung des Toleranz-Dialogs, Teil II: Angewandte Interkulturalität (S. 431-452). Nordhausen: Traugott Bautz.

Frech, S. \& Grimm, D. (Hrsg.), Politische Psychologie und politische Bildung. Schwalbach: Wochenschau-Verlag, Schwalbach.

Friedman, M. (2010). Posttraumatic and Acute Stress Disorder (5. Auflage). Sudbury: Jones Bartlett.

Füllgrabe, U. (1999): Survivability: Überlebensfaktoren in gefährlichen Situationen. Zur Psychologie der Eigensicherung. Praxis der Rechtspsychologie, 9(1), 28-52.

Füllgrabe, U. (2003). Akutes Risiko oder leere Drohung?. Wissenschaftlich fundierte Gefahreneinschätzung von Gewaltandrohungen. Reportpsychologie, 28(2/2003), 150-161.

Gilbert, D., Fiske S. \& Lindzey, G. (Hrsg.). Handbook of social psychology (4. Auflage). New York: Oxford University Press.

Greitemeyer, T., Fischer, P., Kastenmüller, A. \& Frey, D. (2006). Civil Courage and Helping Behavior: Differences and Similarities. European Psychologist, 11(2), 90-98.

Jonas, K. \& Brandstätter, V. (2004). Zivilcourage: Definition, Befunde und Maßnahmen. Zeitschrift für Sozialpsychologie, 35(4), 185-200.

Jonas, K., Boos, M. \& Brandstätter V. (Hrsg.) (2007). Zivilcourage trainieren!, Theorie und Praxis. Hogrefe, Göttingen, 2007.

Kelle, U. \& Kluge S. (2010). Vom Einzelfall zum Typus: Fallvergleich und Fallkontrastierung in der qualitativen Sozialforschung (2., überarbeitete Auflage). Wiesbaden: VS Verlag für Sozialwissenschaften.

Kersten, J. (2008). Medien und Innere Sicherheit. In H.-J. Lange, H. Ohly \& J. Reichertz (Hrsg.), Auf der Suche nach neuer Sicherheit: Fakten, Theorien und Folgen (S. 293-305). Frankfurt am Main: VS Verlag für Sozialwissenschaften.

Labuhn, A. (2004). Zivilcourage: Inhalte, Determinanten und ein erster empirischer Zugang. Frankfurt am Main: Verlag für Polizeiwissenschaft.

Labuhn, A., Wagner, U., van Dick, R. \& Christ, O. (2004). Determinanten zivilcouragierten Verhaltens: Ergebnisse einer Fragebogenstudie. Zeitschrift für Sozialpsychologie, 35(2), 93-103.

Lange, H.-J., Ohly, P. \& Reichertz, J. (Hrsg.) (2008). Auf der Suche nach neuer Sicherheit: Fakten Theorien und Folgen. Frankfurt am Main: VS Verlag für Sozialwissenschaften.

Latané, B. \& Darley, J. (1969). Bystander "Apathy”. American Scientist, 57(2), 244-268.

Latané, B. \& Darley, J. (Hrsg.) (1970): The unresponsive bystander: Why doesn't he help? New York: Appleton-Century-Crofts; zitiert nach: Fischer, P., Greitemeyer, T., Pollozek, F. \& Frey, D. (2006). The unresponsive bystander: Are bystanders more responsive in dangerous emergencies? European Journal of Social Psychology, 36, 267-278.

Latané, B. \& Darley, J. (1976). Help in a crisis: Bystander Response to an emergency. Morristown: General Learning Press; zitiert nach: Bierhoff, H.-W. (2004). Handlungsmodelle für Zivilcourage. In G. Meyer, U. Dovermann, S. Frech \& G. Gugel (Hrsg.): Zivilcou rage lernen: Analysen - Modelle-Arbeitshil- 
fen (S. 60-69). Bundeszentrale für politische Bildung, Landeszentrale für politische Bildung Baden-Württemberg.

Lünse, D. (2006). Zivilcourage: Eine individuelle Tugend. In E. Brix, J. Nautz \& K. Thien (Hrsg.), Zivilcourage (S. 19-58). Wien: Passagen.

Maurer, M. \& Reinemann, C. (2006). Medieninhalte: Eine Einführung. Wiesbaden: VS Verlag für Sozialwissenschaften.

Mayring, P. (2002). Einführung in die qualitative Sozialforschung: Eine Anleitung zum qualitativen Denken (5. Auflage). Weinheim: Beltz.

Meyer, G., Dovermann, U., Frech, S. \& Gugel G. (Hrsg.) (2004). Zivilcourage lernen. Analysen - Modelle - Arbeitshilfen. Bundeszentrale für politische Bildung, Landeszentrale für politische Bildung Baden-Württemberg.

Meyer, G. (2004a). Lebendige Demokratie: Zivilcourage und Mut im Alltag, Forschungsergebnisse und Praxisperspektiven. BadenBaden: Nomos.

Meyer, G. (2004b). Was heißt mit Zivilcourage handeln? In G. Meyer, U. Dovermann, S. Frech \& G. Gugel (Hrsg.): Zivilcourage lernen: Analysen - Modelle - Arbeitshilfen (S. 21-41). Bundeszentrale für politische Bildung, Landeszentrale für politische Bildung Baden-Württemberg.

Meyer, G. (2004c). Vorwort. In G. Meyer, U. Dovermann, S. Frech \& G. Gugel (Hrsg.): Zivilcourage lernen: Analysen - Modelle Arbeitshilfen (S. 8-12). Bundeszentrale für politische Bildung, Landeszentrale für politische Bildung Baden-Württemberg.

Nunner-Winkler, G. (2007). Zum Begriff Zivilcourage. In K. Jonas, M. Boos \& V. Brandstätter (Hrsg.), Zivilcourage trainieren! Theorie und Praxis (S. 21-31). Göttingen: Hogrefe.

Ostermann, Ä. (2004). Zivilcourage und Demokratie. In G. Meyer, U. Dovermann, S. Frech \& G. Gugel (Hrsg.): Zivilcourage lernen: Analysen - Modelle - Arbeitshilfen (S. 5159). Bundeszentrale für politische Bildung, Landeszentrale für politische Bildung BadenWürttemberg.

Oßwald, S., Frey, D., Greitemeyer, T. \& Fischer, P. (2007). Erarbeitung eines Prozessmodells für Zivilcourage. In S. Frech \& D. Grimm (Hrsg.), Politische Psychologie und politische Bildung (S. 114-138). Schwalbach: Wochenschau-Verlag, Schwalbach.

Oßwald, S., Greitemeyer, T., Fischer, P. \& Frey, D. (2010). What is Moral Courage? Definition, Explication and Classification of a Complex Construct. In C. Pury \& S. Lopez (Hrsg.), Psychology of Courage (S. 149-164). Washington: APA.

Piliavin, J. \& Piliavin, I. (1972). Effect of blood on reactions to a victim. Journal of Personality and Social Psychology, 23(3), 353-361.

Polk, K. (1994). When Men Kill: Scenarios of Masculine Violence. Melbourne: Cambridge University Press.

Pury C. \& Lopez S. (Hrsg.), Psychology of Courage. Washington: APA.

Strafgesetzbuch in der Fassung der Bekanntmachung vom 13. November 1998 (BGBL. S. 3322), das durch Artikel 4 des Gesetzes vom 23. Juni 2011 (BGBL. I S. 1266) geändert worden ist.

Wolfgang, M. (1958). Patterns in criminal homicide. Oxford/England: University of Pennsylvania Press; zitiert nach: Polk, K. (1994): When Men Kill. Scenarios of Masculine Violence. Melbourne: Cambridge University Press.

Wolfgang, M. (1970). Victim-Precipitated Criminal Homicide. In M. Wolfgang, L. Savitz \& N. Johnston (Hrsg.), The Sociology of crime and delinquency (2. Auflage, S. 569-578). New York: Wiley.
Wolfgang, M., Savitz, L. \& Johnston, N., (Hrsg.) (1970). The sociology of crime and delinquency (2. Auflage). New York: Wiley.

Yousefi, R. H. \& Fischer, K. (Hrsg.) (2004). Interkulturelle Orientierung - Grundlegung des Toleranz-Dialogs, Teil II: Angewandte Interkulturalität. Nordhausen: Traugott Bautz.

Zimbardo, P. \& Gerrig, R. (2008). Psychologie (18., aktualisierte Auflage). München: Pearson Studium.

\section{sowie folgende Internetquellen:}

Bundesministerium des Innern (Hrsg.) (2011). Kurzbericht der Innenministerkonferenz zur Polizeilichen Kriminalstatistik 2010. Berlin, http://www.bka.de/nn_193236/DE/Publikationen/PolizeilicheKriminalstatistik/IMK Kurzberichte/imkKurzberichte_node.html? nnn=true, [08.12.2011].

Rüster, K. H. (2009). Zivilcourage: ein Thema, das uns alle angeht: Vogel-StraußSyndrom für einen modernen Rechtsstaat nicht hinnehmbar. Medien-Info des WEISSEN RINGS, https://www.weisser-ring.de/ fileadmin/content/presse/medienservice/ Medieninfos/zivi.pdf, [08.12.2011].

Polizeiliche Kriminalprävention der Länder und des Bundes (ProPK), http://www.aktion-tuwas.de/so-kann-ich-helfen/gefahrlos-handeln, [08.12.2011].

\section{Fußnoten}

1 Franca Magnanis: "Je mehr Bürger mit Zivilcourage ein Land hat, desto weniger Helden wird es einmal brauchen.", zitiert nach: Ostermann, 2004, S. 52.

2 Nach Labuhn beschäftigt sich die bisherige Forschung fast ausschließlich mit der Frage danach, „ob“ in Notsituationen eingegriffen wurde. Die Frage nach dem „wie“, also der Art und Weise der Hilfeleistung, wurde bei den meisten Studien nicht beachtet; vgl. Labuhn, 2004, S. 85.

3 Neben der Wahrnehmung des Ereignisses und der Klassifizierung als Notlage müssen das Erkennen und die Übernahme der eigenen Verantwortlichkeit, die Überprüfung der eigenen Handlungsfähigkeit und die Entscheidung über die Art der Hilfeleistung im Rahmen der fünf Stufen des Prozessmodells erfolgreich für eine Hilfeleistung durchlaufen werden. Vgl. Latané \& Darley, 1970; zitiert nach: Bierhoff, 2010, S. 117 ff., Labuhn, 2004, S. 83 ff., Oßwald et al., 2007, S. $131 \mathrm{ff}$

4 Latané und Darley wiesen den Effekt in vier Experimenten nach, wobei sie zwei Prozesse für die verringerte Wahrscheinlichkeit des Eingreifens für ausschlaggebend erachteten. Einerseits das Nichteingreifen anderer, das die Situation insgesamt weniger ernst erscheinen lässt, sowie den Effekt der „Verantwortungsdiffusion"; mehr dazu: Latané \& Darley, 1969, S. 252

5 In Anlehnung an die vorherrschende Meinung gängiger Literatur wird im Weiteren synonym die Bezeichnung „negative Kosten“ verwendet; vgl. Oßwald, Greitemeyer, Fischer \& Frey, 2010, S. 150. Diese können zwischen geringen finanziellen Verlusten durch investierte Zeit oder gespendetes Geld und Erdulden von Beleidigungen, Bedrohungen oder Angriffen durch einen Täter variieren; vgl. Greitemeyer, Fischer, Kastenmüller \& Frey, 2006, S. 90 f.

6 Im Sinne von Beleidigungen, Angriffen usw.; also Handlungen/Konsequenzen, die von anderen Personen initiiert werden.

7 Hilfeleistungssituationen zeichnen sich durch eine dyadische Konstellation aus. Die Interaktion vollzieht sich ausschließlich zwischen Helfer und Opfer.

8 Ein Beispiel für eine triadische Situation ohne anwesendes Opfer stellen diskriminierende Äu- ßerungen dar; vgl. Jonas \& Brandstätter, 2004, S. 188.

9 Akute Notsituationen, die Hilfeleistung bzw. Zivilcourage erfordern, sind durch folgende von Latané \& Darley entwickelten fünf Kriterien gekennzeichnet: „geringe Wahrscheinlichkeit des Auftretens der Notsituation; große Unterschiedlichkeit der bedrohlichen Situation; ein unvorhersehbares Ereignis; Bedrohung für Leben und Wohlbefinden oder Schädigung von Eigentum; Notwendigkeit schnellen Eingreifens"; vgl. Latané \& Darley, 1976; zitiert nach: Bierhoff, 2004, S. 61.

10 Beispiel hierfür sind fremdenfeindliche Diskussionen oder Gespräche, die Diskriminierung einzelner Personen oder Gruppen beinhalten, z. B. am Arbeitsplatz; vgl. Meyer, 2004c, S. ${ }^{\circ} 10$, ebenso Meyer, 2004a, S. 24.

11 Werte wie Menschenwürde, Gleichheit, Rechtstaatlichkeit, Toleranz, soziale Verantwortung sowie die Ablehnung psychischer oder physischer Gewalt

12 Diese ergibt sich aus dem Rückschluss des \323 c Strafgesetzbuch - Unterlassene Hilfeleistung. Die Strafbarkeit einer unterlassenen Hilfeleistung ist vom Erfordernis sowie den spezifischen psychischen und physischen Konstitution abhängig.

13 Vgl. Strafgesetzbuch, $\mathbb{S} 323$ c (Unterlassene Hilfeleistung): „Wer bei Unglücksfällen oder gemeiner Gefahr oder Not nicht Hilfe leistet, obwobl dies erforderlich und ihm den Umständen nach zuzumuten, insbesondere ohne erhebliche eigene Gefahr und obne Verletzung anderer wichtiger Pflichten möglich ist, wird mit Freiheitsstrafe bis zu einem Jahr oder mit Geldstrafe bestraft."

14 Da sich die Studie mit den jeweiligen Einzelfallanalysen auf Inhalte behördlicher Akten bezog, sind zum Schutz der Persönlichkeitsrechte der jeweils Betroffenen keinerlei personenbezogene Daten in den Ausführungen enthalten. Generell lässt das Bayerische Datenschutzgesetz (BayDSG) den Zugriff auf personenbezogene Daten sowie eine Nutzungsänderung für Forschung und wissenschaftliche Zwecke zu, wenn das Forschungsinteresse das Interesse des Betroffenen an seiner Privatsphäre erheblich überwiegt.

15 Eine genauere Differenzierung der Schäden unterblieb, da aus Sicht der Helfer eine detaillierte Beurteilung der dem Opfer entstandenen Schmerzen, Verletzungen usw. aufgrund räumlicher Distanz, Blickwinkel und Fachkenntnis ohnehin nicht möglich war.

16 Die Anzahl der Opfer ergibt sich aus der Gesamtzahl von 32 untersuchten Sachverhalten mit je einem Opfer sowie zwei Vorfällen bei denen der ursprüngliche Helfer selbst Opfer wurde und ein „neuer“ Zeuge einschritt.

17 Eindeutige Verletzungszeichen erhöhen generell die Wahrscheinlichkeit einer richtigen Wahrnehmung des Ereignisses sowie Interpretation als Notlage im Sinne des Prozessmodells zu $\mathrm{Zi}$ vilcourage.

18 Der Ausdruck „victim precipitation“ zielt darauf ab, dass dem Opfer eine eigene, maßgebliche Rolle als - ungewollter, plötzlicher - handelnder Beteiligter in der gesamten Situation zukommt. Das Opfer kann durch das eigene Verhalten selbst die Hauptursache für den eigenen Schaden darstellen. Obwohl Wolfgang in seiner Untersuchung rein verbale Auseinandersetzungen als kausale Ursache der untersuchten Tötungsdelikte ausschloss, ist die Theorie dennoch für die Erklärung eskalationsfördernder Prozesse bei Zivilcourage-Einschreiten nutzbar. 